\title{
Novel SCAMPs Lacking NPF Repeats: Ubiquitous and Synaptic Vesicle-Specific Forms Implicate SCAMPs in Multiple Membrane- Trafficking Functions
}

\author{
Rafael Fernández-Chacón and Thomas C. Südhof \\ Center for Basic Neuroscience, Department of Molecular Genetics, and Howard Hughes Medical Institute, The University \\ of Texas Southwestern Medical Center, Dallas, Texas 75390-9111
}

In vertebrates, secretory carrier membrane proteins (SCAMPs) 1-3 constitute a family of putative membrane-trafficking proteins composed of cytoplasmic N-terminal sequences with NPF repeats, four central transmembrane regions (TMRs), and a cytoplasmic tail. SCAMPs probably function in endocytosis by recruiting $\mathrm{EH}$-domain proteins to the N-terminal NPF repeats but may have additional functions mediated by their other sequences. We now demonstrate that SCAMPs form a much larger and more heterogeneous protein family than envisioned previously, with an evolutionary conservation extending to invertebrates and plants. Two novel vertebrate SCAMPs (SCAMPs 4 and 5), single SCAMP genes in Caenorhabditis elegans and Drosophila melanogaster, and multiple SCAMPs in Arabidopsis thaliana were identified. Interestingly, the novel SCAMPs 4 and 5 lack the N-terminal NPF repeats that are highly conserved in all other SCAMPs. RNA and Western blotting experiments showed that SCAMPs 1-4 are ubiquitously coexpressed, whereas SCAMP 5 is only detectable in brain where it is expressed late in development coincident with the elaboration of mature synapses. Immunocytochemistry revealed that SCAMP 5 exhibits a synaptic localization, and subcellular fractionations demonstrated that SCAMP 5 is highly enriched in synaptic vesicles. Our studies characterize SCAMPs as a heterogeneous family of putative trafficking proteins composed of three isoforms that are primarily synthesized outside of neurons (SCAMPs 2-4), one isoform that is ubiquitously expressed but highly concentrated on synaptic vesicles (SCAMP 1), and one brain-specific isoform primarily localized to synaptic vesicles (SCAMP 5). The conservation of the TMRs in all SCAMPs with the variable presence of $\mathrm{N}$-terminal NPF repeats suggests that in addition to the role of some SCAMPs in endocytosis mediated by their NPF repeats, all SCAMPs perform a "core" function in membrane traffic mediated by their TMRs.

Key words: synaptic vesicles; exocytosis; endocytosis; clathrin; transport vesicle budding; intersectin; EH domain
Secretory carrier membrane proteins (SCAMPs) were discovered as secretory vesicle components in exocrine glands and later shown to be ubiquitous proteins (Cameron et al., 1986; Brand et al., 1991; Laurie et al., 1993). Of the three currently known SCAMPs (SCAMPs 1-3), SCAMP 1 is the most abundant variant (Brand and Castle, 1993; Singleton et al., 1997). The three SCAMPs share a common domain structure composed of a cytoplasmic $\mathrm{N}$-terminal domain with multiple NPF repeats, four highly conserved transmembrane regions (TMRs), and a short cytoplasmic C-terminal tail. SCAMPs are present not only on secretory organelles involved in regulated exocytosis, such as exocrine secretory granules, but also on recycling vesicles that shuttle to and from the plasma membrane in all cells studied (Brand et al., 1991; Brand and Castle, 1993). In addition, SCAMP 1 is highly enriched in synaptic vesicles (Brand et al., 1991).

The ubiquitous distribution of SCAMPs in transport vesicles suggests a fundamental function in vesicular traffic. In support of this hypothesis, five recent findings have suggested a general function for SCAMPs in clathrin-mediated vesicle budding during endocytosis (Fernández-Chacón et al., 2000). (1) In many proteins, $\mathrm{NPF}$ repeats are binding sites for EH-domain proteins such as

Received March 14, 2000; revised Aug. 10, 2000; accepted Aug. 10, 2000.

This study was supported by a postdoctoral fellowship from the Spanish Ministry of Education and Culture and the Fulbright Commission to R.F.-C. We would like to thank Dr. S. Butz (Dallas, TX) and Drs. N. Brose and R. Jahn (Göttingen, Germany) for the gift of antibodies and purified synaptic vesicles, Dr. D. Castle (Charlottesville, VA) for SCAMP monoclonal antibodies, Dr. S. Sugita (Dallas, TX) and Dr. R. Schneggenburger (Göttingen, Germany) for rat brain samples, Dr. J. Albanesi (Dallas, TX) for purified chromaffin granules and adrenal microsomes, and Dr. R. Janz (Dallas, TX) for help with the immunocytochemistry and subcellular fractionation.

Correspondence should be addressed to Dr. T. C. Südhof, Center for Basic Neuroscience, The University of Texas Southwestern Medical Center, 6000 Harry Hines Boulevard, Dallas, TX 75390-9111. E-mail: Tsudho@mednet.swmed.edu.

Copyright (C) 2000 Society for Neuroscience $0270-6474 / 00 / 207941-10 \$ 15.00 / 0$
Eps15 and intersectin (De Beer et al., 1998; Paoluzi et al., 1998). Because most currently known EH-domain proteins are involved in clathrin-mediated vesicle budding from the plasma membrane or trans-Golgi complex, the presence of NPF repeats in the $\mathrm{N}$-terminal region of SCAMPs agrees well with a function in binding EH domains during endocytosis. (2) Biochemical studies confirmed that the N-terminal NPF repeats of SCAMP 1 bind to at least two EH-domain proteins with high affinity, intersectin/EHSH that is involved in endocytosis (Hussain et al., 1999; Okamoto et al., 1999; Sengar et al., 1999), and $\gamma$-synergin that functions in vesicle budding from the Golgi complex (Page et al., 1999). (3) Transfections demonstrated that N-terminally truncated SCAMP 1 acts as a dominant-negative mutant in endocytosis (FernándezChacón et al., 2000). Because SCAMPs 2 and 3 similar to SCAMP 1 also contain N-terminal NPF repeats, it seems likely that they perform an analogous function. (4) Analysis of a mouse knock-out of SCAMP 1 revealed that SCAMP 1 is not essential for survival or for fundamental brain functions, despite the fact that SCAMP 1 is the most abundant SCAMP isoform and the only known SCAMP of synaptic vesicles (Fernández-Chacón et al., 1999). However, detailed analyses of the SCAMP 1 knock-out mice using capacitance measurements in mast cells uncovered a mild phenotype compatible with a function in endocytosis or in membrane fusion. This supports a general role for SCAMPs in membrane traffic but also suggested that there may be redundancy among various SCAMP isoforms. (5) A function for SCAMPs in endocytosis was also indicated by the tyrosine phosphorylation of SCAMPs 1 and 3 by the EGF receptor in fibroblasts (Wu and Castle, 1998), which is similarly observed for other proteins involved in endocytosis such as Eps15 (Fazioli et al., 1993).

On the basis of these findings, a function for SCAMPs in endocytosis, mediated by the N-terminal NPF repeats, appears likely. However, the four conserved TMRs in SCAMPs suggest 
that SCAMPs perform other functions in addition to nucleating clathrin-coat assembly because this assembly would presumably not require multiple TMRs. Furthermore, the lack of a major phenotype in SCAMP 1 knock-out mice, especially in brain in which SCAMP 1 is the dominant isoform, raises the possibility that other currently unrecognized SCAMPs may be present. To address these questions, we have systematically investigated the complement and evolutionary conservation of SCAMPs by the use of data bank searches, molecular cloning, biochemistry, and immunocytochemistry. Data bank searches and cDNA cloning uncovered a new class of SCAMP proteins in vertebrates comprised of two novel isoforms, as well as SCAMP isoforms in Caenorhabditis elegans, Drosophila melanogaster, and Arabidopsis thaliana. These data suggest that all multicellular eukaryotes have SCAMPs. The new class of vertebrate SCAMPs differs from the previously characterized SCAMPs in that the new SCAMPs lack N-terminal NPF repeats and are thus unlikely to function in endocytosis. We also demonstrate that SCAMP 5 is detectably expressed only in brain where it is highly enriched in synaptic vesicles, suggesting that synaptic vesicles contain two SCAMPs with different functional properties.

\section{MATERIALS AND METHODS}

Data bank searches, cDNA cloning, and sequence analyses. GenBank was searched by the use of the BLAST programs of the National Center for Biotechnology Information (NCBI, Bethesda, MD) with the amino acid sequence of SCAMP 1 (Brand and Castle, 1993). Five classes of SCAMPlike sequences were identified in human and mouse expressed sequence tag (EST) databases: SCAMP 1, orthologs of human SCAMPs 2 and 3 that were described during the course of the present study (Singleton et al., 1997), and two novel SCAMPs that we called SCAMPs 4 and 5. To characterize the full-length structures of the various SCAMPs, EST clones were obtained from the IMAGE consortium [human clones \#187735 (SCAMP 2) and \#182330 (SCAMP 3) and mouse clones \#697912 \#423042, \#608076, \#575301, \#608076, \#315146, and \#535376 (SCAMP 2), \#373999 (SCAMP 3), \#442261 (SCAMP 5), and \#572774, \#388542, and \#718670 (SCAMP 4)], mapped with restriction endonucleases, and sequenced. The sequences showed that the mouse SCAMP 2-5 clones \#697912, \#373999, \#442261, \#572774, \#388542, and \#718670 likely contained the full-length coding region whereas the other EST clones were partial. To determine whether the various SCAMPs were conserved in rat, our preferred biochemical model organism, we screened a rat brain $\lambda \mathrm{ZAP}$ cDNA library (Stratagene) with the $1.0 \mathrm{~kb}$ Not I/EcoRI fragment from clone \#187735 (SCAMP 2), the $0.7 \mathrm{~kb}$ NotI/EcoRI fragment from clone \#182330 (SCAMP 3), and the 650 bp EcoRI/Pst I fragment from clone \#442261 (SCAMP 5). $\lambda$ ZAP cDNA clones were plaque-purified and sequenced after in vivo excision using standard procedures (Sambrook et al., 1989). We isolated five different cDNA clones for SCAMP 2, three of which (pSCAMP2-2, -9, and -80) included the coding region from residue 21 to the stop codon and part of the $3^{\prime}$-untranslated region (UTR). For SCAMP 3, we isolated three different clones representing the C-terminal part of the protein; one of them (pSCAMP3-19) included residue 123 to the stop codon and the $3^{\prime}$-UTR. For SCAMP 5, we isolated one full-length clone (pSCAMP5-10) that included $266 \mathrm{bp}$ of $5^{\prime}-\mathrm{UTR}$ and $178 \mathrm{bp}$ of $3^{\prime}$-UTR and a second clone (pSCAMP5-12) containing the coding region starting at residue 28 to the stop codon and the $3^{\prime}$-UTR. In addition to the vertebrate SCAMPs, the data bank searches also identified single SCAMP homologs in Drosophila and C. elegans. Drosophila cDNAs were obtained as EST clones from the IMAGE consortium (clones LD11375, LD15690, and LD14374), of which LD11375 was completely sequenced and found to be full-length. Furthermore, several plant homologs of SCAMPs were identified in $A$. thaliana and Pisum sativum genomic and cDNA sequences in GenBank by BLAST searches. The accession numbers of the genomic $A$. thaliana sequences are AC002560, AC007259, AC006234, and AC002294 and of the $P$. sativum cDNA sequence is AF018093. Sequence analyses were performed using DNA-STAR; alignments were optimized manually. Data bank searches were initially executed using BLAST (Altschul et al., 1997 ) with the default settings of NCBI (for GenBank), the Sanger Center (for the C. elegans database), and the Berkeley Drosophila Genome Project (for the Drosophila database) and the Netscape 3.0 browser. All sequences have been submitted to the GenBank (accession \#AF241833, \#AF241834, \#AF295402, \#AF295403, \#AF295404, AF295405, \#AF295102, and \#AF240784).

Vector construction. To generate the SCAMP 1 expression vector $\mathrm{pCM}$ VSCAMP1, the SCAMP 1-coding region was amplified by PCR from total rat brain cDNA and cloned into the EcoRI site of pCMV5. pCMV-mycSCAMP2 was obtained by PCR amplification of the mouse SCAMP 2-coding sequence with the IMAGE EST clone \#697912 as a template and cloning the product into the EcoRI/ClaI site of pCM V5-myc; pCMV-mycSCAMP3 was constructed by cloning the $1.5 \mathrm{~kb}$ EcoRI/HindIII fragment from the mouse IMAGE cDNA clone \#373999 into pCMV5-myc pCM V-myc-SCAMP5 was generated by PCR amplification of the SCAMP 5 -coding region with the rat brain cDNA clone pSCAMP5-10 as a template and cloning the product into the EcoRI/BamHI site of pCMV5-myc, and pCMV-myc-SCAMP4 was obtained by PCR amplification of the SCAMP4-coding region with the IMAGE mouse cDNA clone \#718670 as a template and cloning the product into the EcoRI/ClaI site of pCMV5myc. All vectors were confirmed by DNA sequencing.

Antibodies. The monoclonal anti-SCAMP antibody SG7C12 was a kind gift of Dr. David Castle (University of Virginia, Charlottesville, VA), and NMDA receptor and synaptic vesicle antibodies were donated by Drs. Nils Brose and Reinhard Jahn (Max Planck Institutes for Experimental Medicine and for Biophysical Chemistry, Göttingen, Germany). Anti-Myc polyclonal antibody was purchased from Upstate Biotechnology (Lake Placid, NY). The anti-SCAMP 1 polyclonal antibody was raised against purified GST-fusion protein of rat SCAMP 1 (residues 1-151) as described previously (Fernández-Chacón et al., 1999). The anti-SCAMP 5 polyclonal antibody was raised against a peptide (sequence, AEKVNNFPPLPKFIPLKPCF YQDF) coupled to keyhole limpet hemocyanin (KLH; Calbiochem) and affinity purified on the peptide coupled to ECH Sepharose (Johnston et al., 1989). To avoid purification of antibodies generated against the cross-linker, coupling to KLH was performed by glutaraldehyde, and coupling to ECH Sepharose (Pharmacia) was by 1-ethyl-3(3dimethylaminopropyl)-carbodiimide hydrochloride.

Subcellular fractionations. Rat brain fractionations were performed basically as described by Huttner et al. (1983) and Butz et al. (1999). Two rat brains were homogenized in $30 \mathrm{ml}$ of buffer A $(0.32 \mathrm{M}$ sucrose, $10 \mathrm{~mm}$ HEPES-NaOH, pH 7.4, $0.1 \mathrm{~mm}$ phenylmethylsulfonyl fluoride, $1 \mathrm{mg} / \mathrm{l}$ pepstatin, $10 \mathrm{mg} / 1$ leupeptin, and $10 \mathrm{mg} / 1$ aprotinin) by the use of a glass Teflon homogenizer (10 strokes; $900 \mathrm{rpm})$. The homogenate was centrifuged at $750 \mathrm{rpm}$ in an HB4 rotor, resulting in pellet $\mathrm{P} 1$ and supernatant that was recentrifuged at $7600 \mathrm{rpm}$ in an $\mathrm{HB} 4$ rotor to yield pellet $\mathrm{P} 2$. P2 was resuspended in $40 \mathrm{ml}$ of buffer $\mathrm{A}$ and recentrifuged at the same speed to yield the synaptosome pellet $\mathrm{P} 2^{\prime}$. The supernatants of the last two spins were pooled ( $\mathrm{S} 2$ and $\mathrm{S} 2^{\prime}$ ). The synaptosomes in $\mathrm{P} 2^{\prime}$ were resuspended in $5 \mathrm{ml}$ of buffer A, lysed hypo-osmotically by dilution with $45 \mathrm{ml}$ of $5 \mathrm{~mm}$ HEPES-NaOH, pH 7.4, containing protease inhibitors (PMSF, leupeptin, pepstatin, and aprotinin), and homogenized with a glass Teflon homogenizer (10 strokes; $900 \mathrm{rpm})$ followed by shaking at $4^{\circ} \mathrm{C}$ for $15 \mathrm{~min}$. The lysed synaptosomes were centrifuged for $20 \mathrm{~min}$ at $10,000 \mathrm{rpm}$ in an HB4 rotor, and the supernatant of this spin was recentrifuged for $1 \mathrm{hr}$ in a TL100.4 rotor at $10,000 \mathrm{rpm}$ to obtain pellet LP1, with the supernatant again centrifuged in the same rotor at $60,000 \mathrm{rpm}$ to generate pellet LP2 and supernatant LS2. Synaptic vesicles purified by controlled pore-glass chromatography (Nagy et al., 1976; Huttner et al., 1983) were a kind gift of Dr. Stefan Butz. Chromaffin granules and adrenal microsomes were prepared by homogenizing bovine adrenal medullae in $5 \mathrm{vol}$ of $0.3 \mathrm{M}$ sucrose and $10 \mathrm{mM}$ HEPES-NaOH, pH 7.4, followed by a 10 min centrifugation at $800 \times g$ to remove debris. The resulting supernatant contains chromaffin granules that were pelleted by centrifugation for $20 \mathrm{~min}$ at $26,000 \times g$ and further purified over a $1.6 \mathrm{M}$ sucrose step gradient (Smith and Winkler, 1967). The microsomes in the supernatant of the $26,000 \times g$ centrif ugation were pelleted by a $1 \mathrm{hr}$ centrifugation at $100,000 \times g$. The protein concentrations of all fractions were determined with the Bio-Rad Coomassie blue protein assay.

Brain immunocytochemistry. Immunocytochemical analysis was performed on cryostat brain sections from perfusion-fixed adult rats. Antibody dilutions were 1:500-1:1000. Antibody reactions were detected by the use of the peroxidase-antiperoxidase technique and heavy metal enhancement as described (Rosahl et al., 1995). As controls for the specificity of the observed staining patterns, sections were stained with the various antibodies in the presence of the antigen used to raise the antibody to block specific reactivity, and sections were stained with preimmune and nonimmune sera.

$R N A$-blotting analyses. These analyses were performed with commercially available rat multitissue RNA blots (Clontech) that were consecutively probed for SCAMPs 1-5 to ensure that the relative expression of SCAMPs in different tissues is comparable. All hybridizations were performed at high stringency $\left(42^{\circ} \mathrm{C}\right.$ overnight in $50 \%$ formamide, $10 \times$ Denhardt's solution, and $0.1 \mathrm{mg} / \mathrm{ml}$ salmon sperm DNA) with uniformly $\left[\alpha-{ }^{32} \mathrm{P}\right] \mathrm{dCTP}-\mathrm{labeled}$ probes. Probes used were the following: a $500 \mathrm{bp}$ EcoRI/HindIII fragment from the $5^{\prime}$-coding region of rat cDNA SCAMP 1, a 600 bp EcoRI fragment from the rat cDNA clone pSCAMP2-2, a 450 bp fragment from the rat cDNA clone pSCAMP3-19, a 650 bp EcoRI/PstI fragment from the IMAGE mouse cDNA clone \#442261 (SCAMP 5), and a 300 bp Pst I/AvaI fragment from the IMAGE mouse cDNA clone \#388542 (SCAMP 4). Filters were washed twice for $30 \mathrm{~min}$ at $65^{\circ} \mathrm{C}$ in $0.2 \times$ SSC and $0.5 \%$ SDS and exposed for 1-5 d.

Cell culture, transfections, and protein analysis. COS cells were cultured in DMEM with $10 \%$ fetal bovine serum and transfected with DEAEdextran with chloroquine and a 2 min glycerol shock (Gorman, 1985). Seventy-two hours after transfection, cells were washed once and harvested in PBS with a rubber policeman. Protein was solubilized by incubation of the cells, at $4^{\circ} \mathrm{C}$ for at least $1 \mathrm{hr}$, in a Ringer's solution containing 2\% 3-([3-cholamidopropyl]dimethylammonio)-1-propanesulfonate. The insoluble fraction was separated by low-speed centrifugation. The supernatant containing protein was mixed with $2 \times$ sample buffer and analyzed by SDS-PAGE electrophoresis. 
Miscellaneous procedures. SDS-PAGE and immunoblotting were performed according to standard procedures (Laemmli, 1970; Towbin et al., 1979).

\section{RESULTS \\ SCAMPs form a large family of evolutionarily conserved membrane-trafficking proteins}

EST data bank searches uncovered two novel SCAMP isoforms in vertebrates in addition to SCAMPs 1-3. To obtain the full-length cDNAs of various SCAMPs and to characterize their protein products, we sequenced multiple mouse and human EST clones encoding SCAMPs 2-5 that were obtained from the IMAGE consortium and constructed expression vectors for these proteins. In addition, we used the EST clones as probes to isolate rat cDNA clones for some of these SCAMPs. These experiments included SCAMPs 2 and 3 that were only reported during the course of the present study when we had characterized them (Singleton et al., 1997). The full-length sequences for mouse and/or rat SCAMPs 1-5 obtained in our studies are aligned with each other in Figure $1 A$, demonstrating a high degree of homology among the various SCAMP isoforms.

In addition to analyzing vertebrate sequences, we also searched data banks for SCAMPs expressed in invertebrates and plants. In the $C$. elegans and Drosophila data banks, these analyses suggested that only a single SCAMP is expressed in these organisms. To evaluate how similar the Drosophila and vertebrate SCAMPs are, we determined the full-length Drosophila SCAMP cDNA sequence (aligned with the vertebrate sequences in Fig. $1 A$ ). Furthermore, the data bank searches uncovered a full-length SCAMP cDNA sequence from a plant, $P$. sativum, and four SCAMPs in the $A$. thaliana genomic sequences. As shown in the alignment (Fig. 1A), analysis of the Arabidopsis sequences suggested that its four SCAMP genes are closely related to each other and to the $P$. sativum sequence and more distantly to the animal sequences. The analyses of the various SCAMP sequences led to a domain model that is shown in Figure $1 B$. According to this model, SCAMPs are composed of an N-terminal region with multiple NPF repeats, four TMRs, and a variable cytoplasmic tail. All of the SCAMPs share this domain organization except for the novel SCAMPs 4 and 5 that lack the N-terminal NPF repeats.

The presence of SCAMPs in vertebrates, invertebrates, and plants supports a role for SCAMPs as universal membranetrafficking proteins in multicellular organisms. The conservation of SCAMP sequences between the various isoforms and organisms is striking and reveals an interesting pattern. The most highly conserved sequences are the TMRs, especially TMRs 2 and 3 and their cytoplasmic connecting loop. By contrast, the intravesicular connecting loops between TMRs exhibit much less similarity (Fig. 1A). The intravesicular loops contain no cysteines or N-glycosylation sites, consistent with biochemical evidence that SCAMPs are not glycosylated or disulfide linked (data not shown). The lowest degree of homology between SCAMPs, however, is observed in the $\mathrm{N}$ - and C-terminal cytoplasmic sequences. Except for the sequences adjacent to the TMRs, the $\mathrm{N}$ - and $\mathrm{C}$-terminal regions exhibit no significant sequence identity. The only conserved feature of the N-terminal sequences is the NPF repeats, but even these are variable because they are absent from the novel SCAMPs 4 and 5 . A special feature of the TMRs in SCAMPs is that they contain a relatively high content of phenylalanine, most strikingly in the beginning of TMR 3 that includes the conserved sequence FxFFxFFFxFF. In addition, several TMRs contain conserved central hydrophilic residues (e.g., asparagine in the middle of TMR 1) suggestive of interactions between TMRs.

\section{Tissue-specific expression of SCAMPs}

To determine which tissues express the various SCAMP isoforms, we used RNA-blotting experiments (Fig. 2). Although we found a large variation in expression levels of individual SCAMPs between tissues, most coexpressed SCAMPs 1-4, whereas SCAMP 5 was detectable only in brain. No mRNA for a SCAMP isoform is uniformly present at the same level in all tissues. For example, heart expresses SCAMPs 1-3 but no SCAMP 5 and little SCAMP 4. Conversely, in brain, mRNAs for SCAMPs 1 and 5 are present at high levels, whereas the other three SCAMPs exhibit low abundance. Overall, these data demonstrate that SCAMPs 1-4 are ubiquitous tissue components whereas SCAMP 5 is the only SCAMP with a highly restrictive expression pattern.

\section{Analysis of the reactivity of SCAMPs with the SCAMP monoclonal antibody}

The initial definition of SCAMPs as a protein family was greatly aided by a monoclonal antibody developed by Castle and colleagues that reacts with multiple SCAMPs on immunoblots (Brand et al., 1991; Singleton et al., 1997). To gain insight into the reactivity of the five rodent SCAMPs that we identified with this monoclonal antibody, we transfected all SCAMPs into COS cells and analyzed them by immunoblotting. To ensure that the recombinant proteins were in fact produced in the transfected cells, we expressed SCAMPs $2-5$ as fusion proteins with an N-terminal myc-tag and confirmed synthesis of the various proteins with a myc-tag antibody.

First we evaluated the presence of SCAMPs in control COS cells and rat brain homogenates (Fig. 3, lanes 1, 2). Two endogenous SCAMPs were recognized by the SCAMP monoclonal antibody in the control COS cells at the level of sensitivity used, whereas only one SCAMP was observed with this antibody in brain. Comparison of the endogenous COS cell and brain SCAMPs with the various transfected samples identified the COS cell SCAMPs as SCAMPs 1 and 2 (Fig. 3) and the brain SCAMP as SCAMP 1. This was also confirmed by immunoblotting with an polyclonal antibody against SCAMP 1 that recognized only this isoform (Fig. 3). We then analyzed COS cells transfected with the various SCAMP expression vectors. Immunoblotting with the myc-epitope antibody confirmed that SCAMPs 2-5 were synthesized in transfected COS cells. Despite identical transfection conditions, the levels of SCAMPs 2 and 3 produced were much higher than those of SCAMPs 4 and 5. Twenty times more COS cell protein had to be loaded after transfection with SCAMPs 4 or 5 than after transfection with SCAMPs 2 and 3 to detect an immunoblotting signal with the myc antibody (Fig. 3, lanes 4-7). As a result, very little of the SCAMP 1-3 COS cell extracts was used, and the endogenous COS cell SCAMPs are not seen in these samples. These are, however, visible in the lanes loaded with the SCAMP 4 and 5 COS cell extracts because 20 -fold more material was loaded. Probing transfected COS cells with the SCAMP monoclonal antibody revealed that it exhibits a high affinity for SCAMPs 1 and 2. Transfected SCAMP 2 is observed as a doublet with the SCAMP antibody, but only the upper band is detected with the myc antibody because in the SCAMP 2 construct, the myc-epitope sequence precedes the endogenous initiator methionine, resulting in the use of two consecutive initiator methionines during translation. Loading larger amounts of the SCAMP 3 sample indicated that SCAMP 3 also reacts with the SCAMP monoclonal antibody, but more weakly than do SCAMPs 1 and 2 (data not shown; see also Fig. $7 B$ below), whereas SCAMPs 4 and 5 were not recognized by the monoclonal antibody under any condition.

\section{The SCAMP 5 sequence is full-length}

The selective lack of NPF repeats only in SCAMPs 4 and 5 is surprising (Fig. 1B). Is it possible that the $5^{\prime}$ end of the sequences of SCAMPs 4 and 5 is incomplete, or do SCAMPs 4 and 5 truly lack NPF repeats and constitute a new class of SCAMPs? The first possibility would support a general function for all SCAMPs in recruiting clathrin coats via EH-domain protein binding to the NPF repeats (Fernández-Chacón et al., 2000). This possibility is consistent with the relatively large mRNA for SCAMP 5 in brain (Fig. 2). The second possibility suggests that the NPF repeats may be peripheral for SCAMP function and that the functionally central part of SCAMPs is their shared sequences, especially the TMRs. To test this question and to generate the reagents for 
$\mathbf{v} \boldsymbol{\nabla}$

$\nabla \nabla$

$\boldsymbol{\nabla v}$ $r$ Sc1

$r$ Sc2

m Sc2

m Sc3

$\mathrm{Dm} \mathrm{Sc}$

$\mathrm{Ce} \mathrm{Sc}$

Ps SC

At SCA

At $S C B$

At $S C C$

At $S C D$

m Sc4

$x$ Sc5

m Sc5

r Sc1

$r \mathrm{Sc2}$

m Sc2

$r \mathrm{Sc} 3$

m Sc3

Dm Sc

Ce Sc

Ps SC

At SCA

At $S C B$

At $\mathrm{SCC}$

At $S C D$

m Sc4

$r$ Sc5

m Sc5

$r$ Se1

$r$ Sc2

m Sc2

I Sc3

m Sc3

Dm Sc

Ce Sc

Ps Sc

At SCA

At $S C B$

At $\mathrm{SCC}$

At $S C D$

m Sc4

$r$ Sc5

m Sc5

$r$ Sc1

$r \mathrm{Sc} 2$

m Sc2

$x \mathrm{Sc} 3$

m $\mathrm{Sc} 3$

Dm Sc

$\mathrm{Ce} \mathrm{Sc}$

Ps SC

At SCA

At $S C B$

At $\mathrm{SCC}$

At $S C D$

m Sc4

$r$ Sc5

Sc 5

r Sc1

$r$ SC2

m Sc2

$r \mathrm{Sc}^{3}$

m Sc3

Dm SC

$\mathrm{Ce} \mathrm{Sc}$

Ps Sc

At $S C A$

At $\mathrm{SCB}$

At $S C C$

At $S C D$

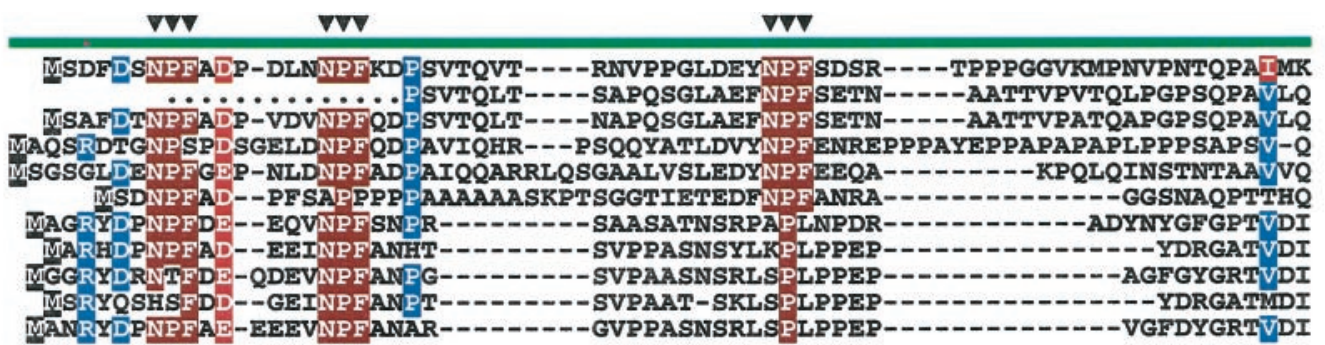

65

44

72

66

54

52

39

51

46

(1)

MAGKENNFPPLPRFIPLK 18

MAEKVNNFP PLPKFIPLK 18

P- - - - - TEEHPAYTQITKEHALAQAELLKRQEELERKAAELDRREREMTQNLS---QHGRINNWPPLPSNFPVG 130

P--.----SVEPAQPSPQAVAAAAQAGLLRQQEELDRKAAELERKERELQNTA-ANLFVRENNWPPLP SWCPVK 110 ------SVEPAQPTPQAVAAAAQAGLLRQQEELDRKAAELFRKERELQNTA-ANLHVRDNNWPPLP SWCPVK 130

SSRKLSPTEPRNYGSYS-TQASAAAATAELLKK

PLSONIPPPQTSSLGASAPSTSIOITSBELQRRQEELDRKAAELDRREOQLOGNV-----PQLNNWP PLPDNFCVK 137

STGSLGNKSAGMDDELFRKQQDLERRAOELRMREEELDRRQR SAAGGNNLNTNAQ--NNAPRPHNWPPLPTIIPIE 128

P---- - - - LDTST---DGKKKERDLQAKEAELRKREQEVRRKEEAIARAG---IVIFEKNWP PF------F 104

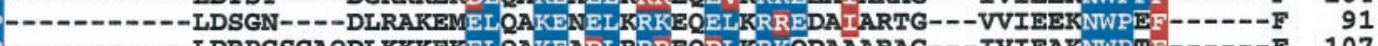

-------LDRPGSGAQDLKKKEKELQAKBADLRRREQDLKRKQDANARAG---IVIEAKNWPTF----- F 107

$\begin{array}{lll} & \end{array}$

$\begin{array}{llll}\text { Cytoplasmic TMR } 1 & \text { Intrav.LOOP } 1 & \text { TMR } 2\end{array}$

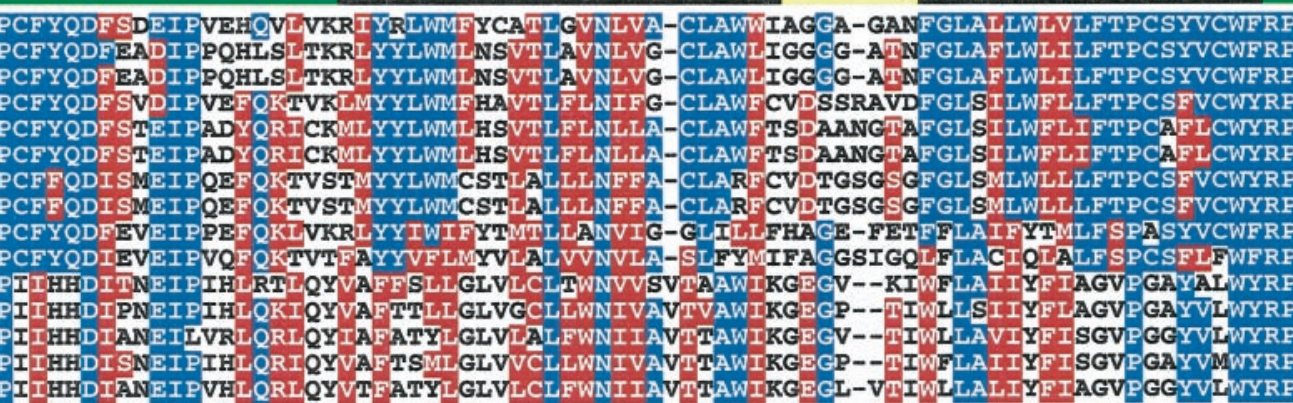

92

92

92
205

185

205

99

222

211

203

178

165

181

172

180

Cytopl. Loop

TMR 3

Intravesicular LoOp 2

TMR 4

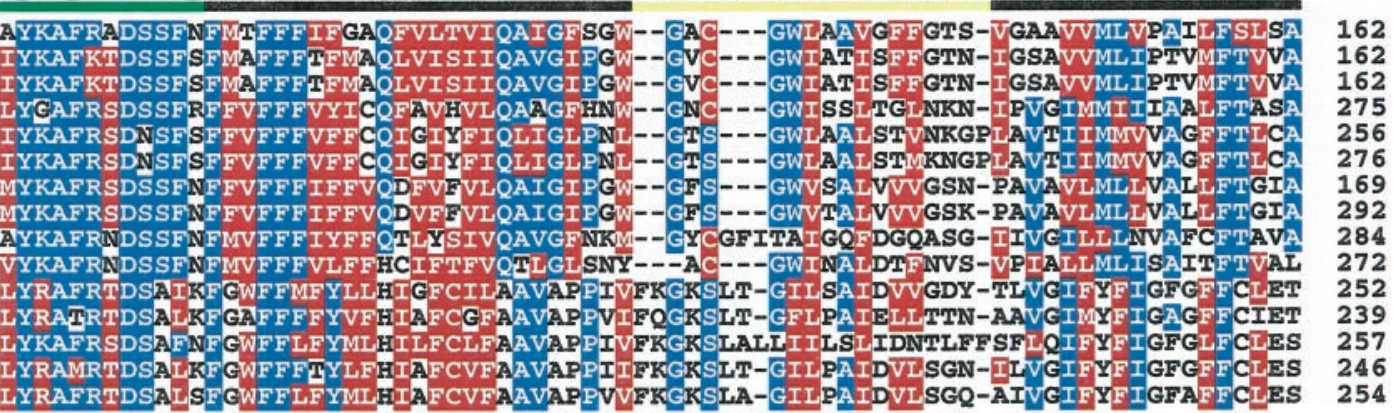

Cytoplasmic

LVMAVTIVKVHRIYRGAGGSLOKAQTEWSA GTWRNPPSREAOFNSFSGNSLPEYPT-VPSYSSSGGHW•

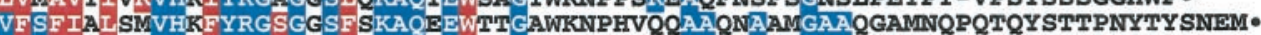

VFSF IAL SMVHKFYRGSGGSF SKAQEEWTT GAWKNPHVQQAAQNA AM GAAQGAMNQPQTQYSATPNYTYSNEM・

I SLVMFKKVHGLYRTTGASFEKAQQEFATGVMSNKTVQTAAANAASTAATSAAQNAFKGNQM•

GLSLFLLQRVHAFYRRTGASFQQAQBEF SQGIFSSRTFRTAASSAAR GFQGN•

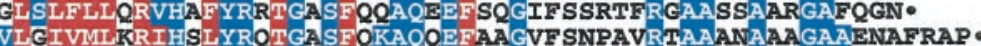

VLGIVMLKR I HSLYRQTGASFQKAQQEFAAGVF SNPAVRTAAANAAA GAAENAFRAP.

VANVLMITKI HSIYR STGA SNAKAQAEFTTEFLRNQHVQWAASSAVNTAINSQFNNSRY•

TGMVTALVKVHRLYRGAGFS IDKARQ BFTNGVMSDAGVQRATQAATQAAAGAAFNQATQGRF.

LISIWVIQQVYMIFER GGGKTA---

LINTWIOOVYAYFRGSGKAA-..--

VVSIWVIQQVYMYFRGSGKAD-.-.-.-- DMRRDAARGAM-RAAI •

VS IWVIQQVYMYFRGSGKAA--

VSIWVIQQVYMYFRGSGKQD-.-...-..-OMRRBAARGAL-RAAV.

230

235

235

338

309

329

226

349

343

334

289

275

293

282
290

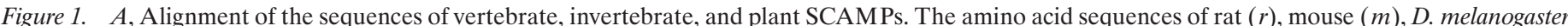

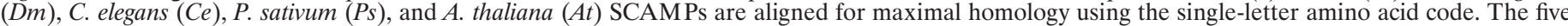

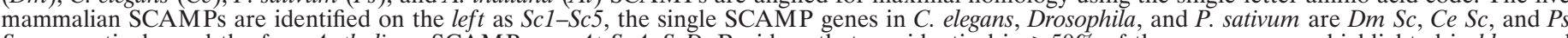

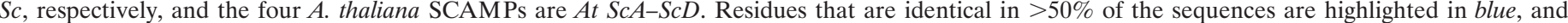

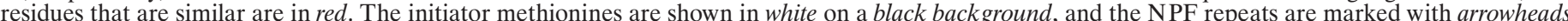

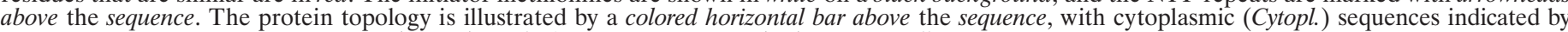

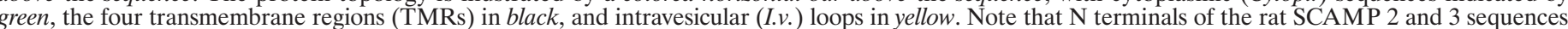

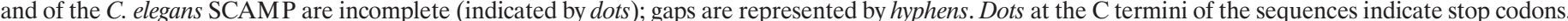

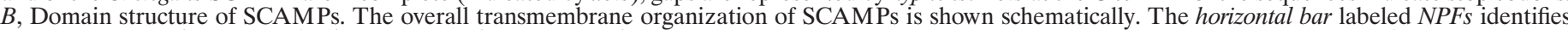

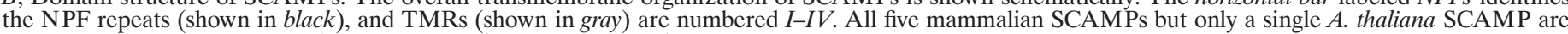

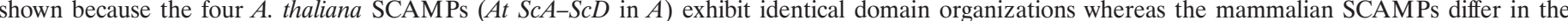
presence or absence of the N-terminal domain containing NPF repeats. a.a., Amino acids. 


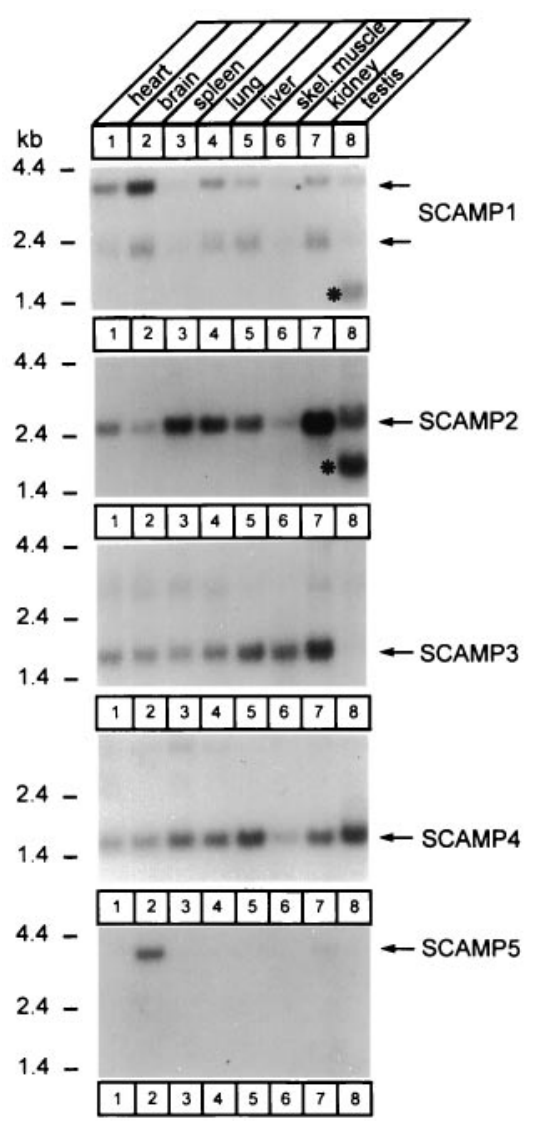

Figure 2. Tissue distribution of expression of SCAMPs $1-5$ analyzed by RNA blotting. An RNA blot containing polyA-enriched RNAs from the indicated rat tissues was hybridized consecutively with cDNA probes from SCAMPs 1-5. Numbers on the left indicate positions of molecular weight markers. The asterisk in the RNA blots for SCAMPs 1 and 2 corresponds to an artifactually hybridizing testis mRNA that is frequently observed with many probes. Note that two mRNA species for SCAMP 1 (arrows) are expressed in most tissues, possibly because of differential polyadenylation. skel., Skeletal.

analyzing the localization of SCAMP 5 in brain, we raised and affinity-purified antibodies to a peptide from SCAMP 5. Immunoblotting revealed that this antibody detects a single band in brain and reacts with SCAMP 5 expressed by transfection in COS cells (Fig. 3, bottom). The SCAMP 5-immunoreactive band was not observed with preimmune serum or after blockage with the peptide used for immunization (data not shown). The size of brain SCAMP 5 protein was slightly smaller than that of myc-tagged SCAMP 5 expressed in COS cells, consistent with addition of the myc-epitope in the recombinant SCAMP 5 (Fig. 3). Thus the SCAMP 5 expression construct encodes the full-length protein, suggesting that the SCAMP 5 sequence is full-length and that SCAMP 5 normally lacks NPF repeats. Although we did not analyze SCAMP 4 in the same manner, its sequence homology to SCAMP 5 suggests that it also lacks NPF repeats and represents the ubiquitous counterpart to neuronal SCAMP 5.

\section{Universal versus brain-specific expression of SCAMP 1 versus SCAMP 5}

The RNA-blotting experiments suggest that SCAMPs 1-4 are ubiquitously expressed whereas SCAMP 5 may be brain specific. This was confirmed in immunoblotting experiments of rat tissues (Fig. $4 A$; data not shown). Interestingly, SCAMP 5 was not only absent from standard non-neuronal tissues such as kidney and lung but also undetectable in neuroendocrine glands that express many other neuron-specific proteins such as the synaptic vesicle proteins synaptophysin and synaptotagmin. We observed no SCAMP 5 in adrenal gland or adenohypophysis in the presence of high levels of
SCAMP 1 in these tissues and of high levels of SCAMP 5 in brain and neurohypophysis (Fig. $4 A$ ). To study the relative distribution of these SCAMPs further, we analyzed multiple cultured cells. In confirmation of the idea that SCAMP 1 is a universal cell component, all cells tested synthesized SCAMP 1, mostly together with SCAMP 2 (Fig. 4B). SCAMP 5, by contrast, was absent except for a very weak signal in ATT20 cells that are transformed hypophyseal cells. Primary astrocytes cultured from rat brain did not express SCAMP 5, indicating that it is not a glial protein. Even cell lines that are thought to be related to neurons, pheochromocytoma 12 (PC12) cells and human HNT neuronal precursor cells, contained no detectable SCAMP 5. This result suggests that SCAMP 5 expression is highly specific for mature brain cells.

We next explored the developmental expression profile of SCAMPs in brain. Immunoblot analysis of total rat brain proteins from different developmental stages revealed that SCAMP 1 is detectable from the earliest embryonic time points analyzed whereas SCAMP 5 cannot be observed until the second week after birth (Fig. 5). The expression profile of SCAMP 5 is similar to that of synaptotagmin I and other synaptic vesicle proteins that become abundant only with the increasing growth of synapses after birth (Fig. 5). The observed differences in relative expression between SCAMPs 1 and 5 were not caused by differences in antibody affinity and the nonlinearity of ECL signals because the same relative expression profiles were observed after loading different amounts of protein per lane (data not shown). The dramatic induction of SCAMP 5 levels in brain during postnatal development supports the idea that SCAMP 5 plays a specialized role in the brain correlated with synapse formation and stabilization but, different from the other SCAMPs, not involved in general ubiquitous functions.

\section{Localization of SCAMPs 1 and 5 in brain}

The restricted expression of SCAMP 5 in brain raises the question whether SCAMP 5 is a general component of all brain areas or a specialized protein present only in some brain regions. To test this, we first analyzed microdissected brain areas by immunoblotting for SCAMPs 1 and 5. All brain areas contained SCAMPs 1 and 5 in a similar ratio, suggesting that both SCAMPs are widespread components of various brain structures (data not shown). We then used immunocytochemistry to localize SCAMPs 1 and 5 in brain. Two brain areas were analyzed: the hippocampus and cerebellum (Fig. 6; data not shown). Specificity of the staining patterns was ascertained by competition with the antigens that were used to generate the respective antibodies and by use of preimmune sera. Staining of hippocampal sections with the SCAMP antibodies revealed a similar but distinct distribution. For both SCAMPs, the staining pattern resembled that of a synaptic vesicle protein, not unexpected for SCAMP 1 because it has been demonstrated to be highly concentrated on synaptic vesicles (Brand and Castle, 1993). The distribution of SCAMP 1 mostly resembled that of synaptoporin (Fig. 6A,E) with a discrete band of synapses labeled in the dentate gyrus and strong labeling of the mossy fiber terminals in the CA3 region. SCAMP 5 in turn was more similar to synapsins that also exhibit a more broad distribution in the dentate gyrus. The similar synaptic distributions of SCAMPs 1 and 5 were confirmed in staining experiments of the cerebellum that supported the idea that the two SCAMPs are expressed in similar patterns in all brain areas, that their staining resembles that of a synaptic vesicle protein, and that there are no large groups of neurons that lack one or the other of the two SCAMPs (data not shown).

\section{SCAMPs 1 and 5 are synaptic vesicle proteins}

The immunocytochemistry results are consistent with a colocalization of SCAMPs 1 and 5 on synaptic vesicles. To test this hypothesis directly, we performed subcellular fractionations. When we studied the relative levels of SCAMPs 1 and 5 in samples containing increasingly purer synaptic vesicles, we observed a significant augmentation of the SCAMP 1 and 5 signals, whereas NMDA 
Figure 3. Analysis of SCAMP antibodies with transfected COS cells. COS cells trans1 ), rat brain homogenate (lane 2), and SCAMP 1-5 expression vectors (lanes 3-7) were analyzed by immunoblotting as indicated on the right. Blots were probed with polyclonal anti-Myc antibodies, monoclonal anti-SCAMP antibody (SG7C12), polyclonal anti-SCAMP 1 antibody (R806), and polyclonal anti-SCAMP 5 antibody (T812). Expression vectors for SCAMPs 2-5 encoded the SCAMP-coding regions with a myc-epitope preceding the endogenous initiator methionine codon to allow monitoring of expression of the transfected proteins. Translation of these cDNAs is initiated from the N-terminal mycepitope methionine codon preceding the coding sequence and from the endogenous methionine immediately after the myc-epitope, resulting in recombinant proteins expressed as doublets as visualized with SCAMP antibodies. Note that very different amounts of material were loaded depending on the expression levels of the various transfected proteins in COS cells (indicated on top) and that nontransfected COS cells normally express SCAMPs 1 and 2 as shown in lane 1. In all blots asterisks in lanes 6 and 7 identify the positions of COS-expressed SCAMP 4 and 5 to facilitate orientation on the gels. Numbers on markers. fected with salmon sperm control DNA (lane the left indicate positions of molecular weight

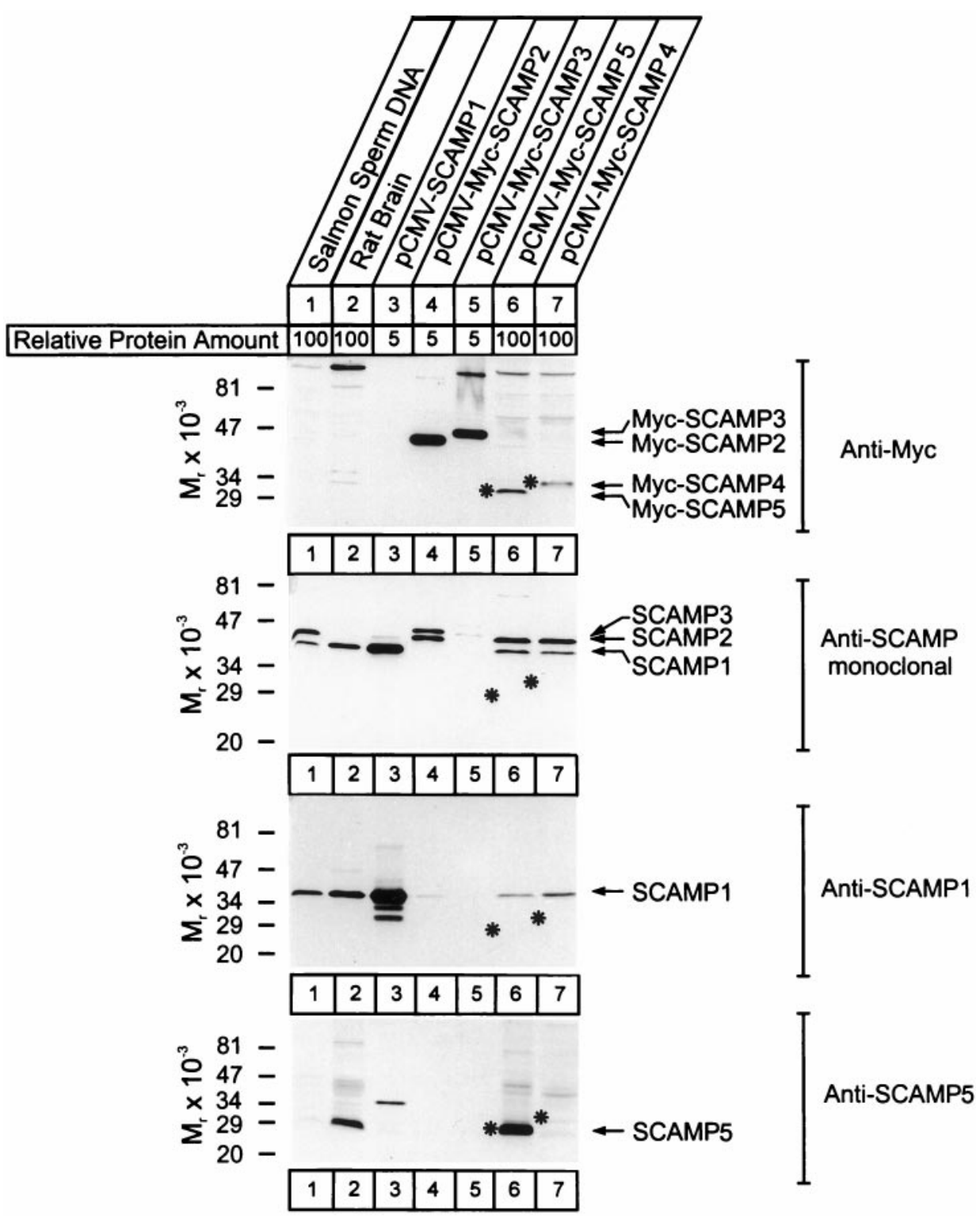

receptors were deenriched (Fig. 7A). Highly purified vesicles obtained by controlled pore-glass chromatography exhibited the strongest signal, demonstrating that both SCAMPs are genuine synaptic vesicle proteins. Interestingly, the relative ratio of SCAMP 5 to 1 increased with progressing purity of the synaptic vesicles, suggesting that SCAMP 5 is more specific for synaptic vesicles than is SCAMP 1. This result is consistent with the ubiquitous distribution of SCAMP 1 in all cells in contrast to the restricted distribution of SCAMP 5 in the brain (Fig. 4B).

Are other SCAMPs present on synaptic vesicles in addition to SCAMPs 1 and 5? The RNA-blotting results showed that SCAMP 1 is most abundant in brain whereas SCAMPs 2-4 have their lowest expression levels in this tissue (Fig. 2), suggesting that SCAMPs 2-4 are unlikely to be synaptic vesicle components. To address this by an independent method, we analyzed relatively large amounts of highly purified synaptic vesicles by immunoblotting with the SCAMP monoclonal antibody in comparison with transfected COS cells to identify the bands observed (Fig. 7B). Only SCAMP 1 was detected in synaptic vesicles. These experiments definitively eliminate the presence of SCAMP 2 on the vesicles but do not exclude the possibility that SCAMP 3 is a minor component of vesicles because SCAMP 3 only weakly reacts with the monoclonal antibody. Furthermore, these experiments do not address SCAMP 4 because we do not have an antibody to that particular SCAMP isoform.

\section{Distribution of SCAMPs in chromaffin granules and microsomes from bovine adrenal gland}

All currently known general synaptic vesicle proteins except for synapsins are also present on the secretory granules of neuroendocrine cells. In addition to secretory granules, neuroendocrine cells contain a population of small vesicles of unknown function, referred to as synaptic-like microvesicles, that are purified with microsomes and are characterized by high concentrations of synaptic vesicle proteins (Fischer von Mollard et al., 1990). To analyze the relative distribution of SCAMPs in the various vesicles, we purified chromaffin granules and microsomes from bovine adrenal medulla and studied their complement of SCAMPs in comparison with that of bovine brain (as a positive species control) and with that of transfected COS cells (to identify the various bands). In confirmation of the results with neuroendocrine cells described above (Fig. 4), SCAMP 5 was absent from all adrenal gland fractions, although SCAMP 1 and synaptic vesicle proteins such as synaptotagmin and synaptophysin were abundantly present (Fig. 8). By contrast, SCAMP 2 was present in the adrenal medulla. SCAMP 2 was almost completely excluded from chromaffin granules but selec- 

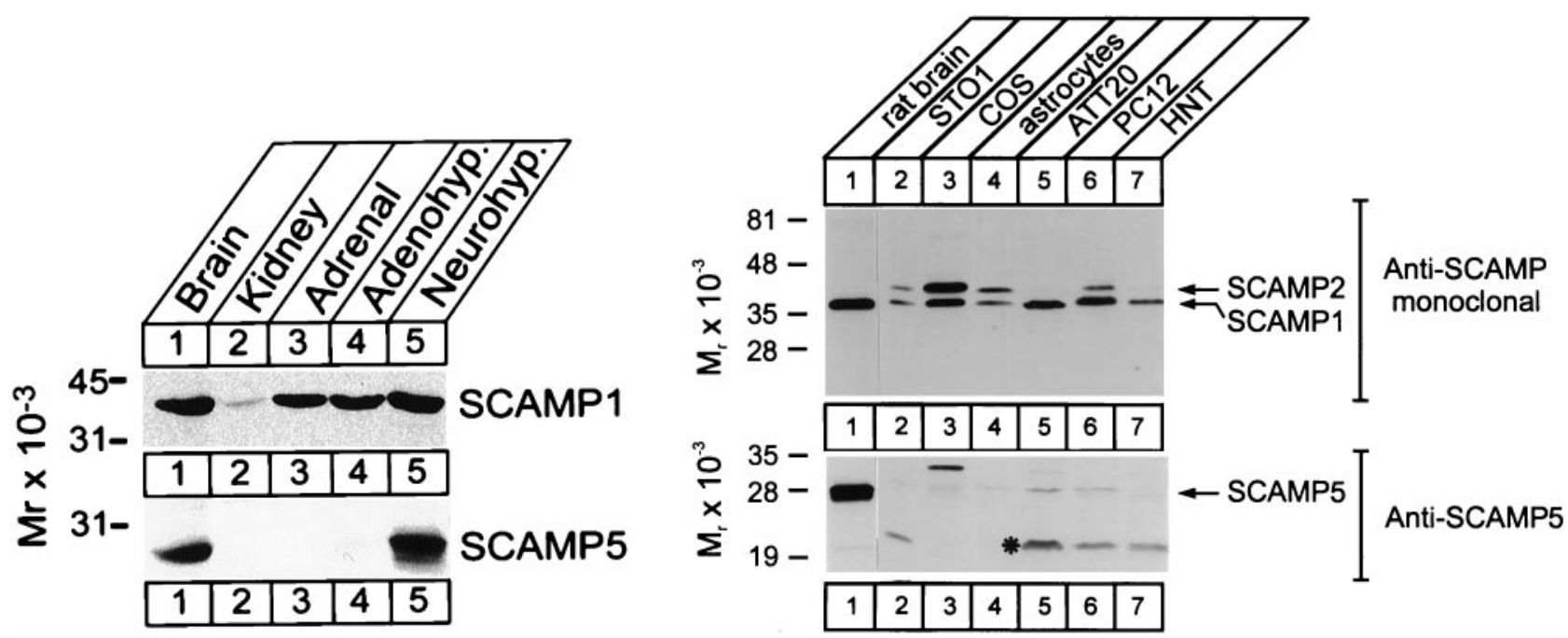

Figure 4. Expression of SCAMPs in endocrine tissues and cell lines. A, Immunoblotting analysis of total protein from the indicated rat tissues (17 $\mu \mathrm{g} /$ lane) with polyclonal antibodies to SCAMPs 1 and 5 as indicated. Adenohyp., Adenohypophysis; Neurohyp., neurohypophysis. B, Comparative immunoblotting analysis of proteins from rat brain homogenate (lane 1) and indicated cell lines (lanes 2-7) with the SCAMP monoclonal antibody and the SCAMP 5-specific polyclonal antibody. The positions of SCAMPs 1 and 2 (recognized by the monoclonal antibody) and SCAMP 5 are indicated on the right, and locations of molecular weight markers are on the left. The asterisk indicates a nonspecific band that cross-reacts with the SCAMP 5 polyclonal antibody in some cell lines but not in brain.

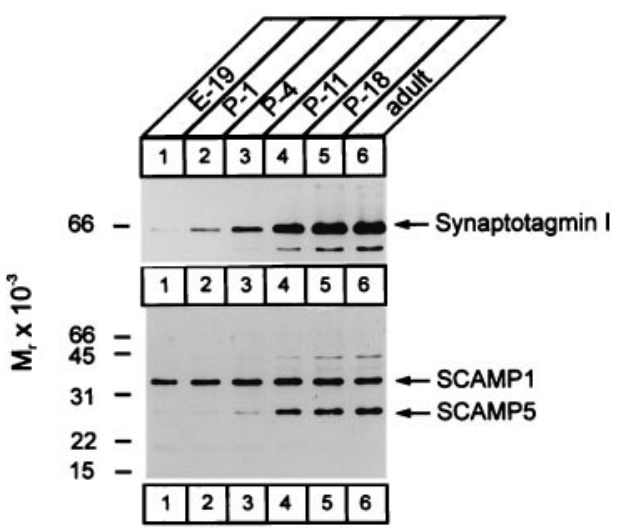

Figure 5. Developmental dynamics of SCAMP 1 and 5 expression in brain. Equal amounts of protein in brain homogenates from rats of the indicated ages were analyzed by immunoblotting for synaptotagmin I (top) and for SCAMPs 1 and 5 (bottom). Numbers on the left indicate positions of molecular weight markers. E-19, Embryonic day $19 ; P-1$ to $P$-18, postnatal days $1-18$.

tively enriched in microsomes, suggesting differential localization of SCAMPs in the same cell type (Fig. 8).

\section{DISCUSSION}

\section{The SCAMP protein family}

Our data characterize SCAMPs as a large, evolutionarily conserved gene family that is probably expressed in all multicellular organisms, but not in yeast. We show that in vertebrates, two classes of SCAMPs are expressed that differ in their domain structures: the traditional SCAMPs composed of an N-terminal region with multiple NPF repeats and four TMRs and a novel class of SCAMPs that lack the N-terminal NPF-repeat domain. Both classes of SCAMPs contain members that are ubiquitous (SCAMPs 1-4) and that are enriched on synaptic vesicles (SCAMPs 1 and 5), suggesting a universal role in membrane traffic at the plasma membrane. Compared with other SCAMPs, SCAMP 5 appears to have a unique specialized synaptic function in mature neurons as suggested by the following evidence: (1) Of all SCAMP isoforms, SCAMP 5 is the only isoform with a restricted brainspecific expression profile. (2) Immunocytochemistry at the light level revealed a localization of SCAMP 5 similar to that of other synaptic vesicle proteins. (3) SCAMP 5 is produced at significant levels only after the second postnatal week, a period of intense synaptogenesis. The developmental expression pattern of SCAMP 5 resembles that of other synaptic vesicle proteins, although SCAMP 5 is expressed even later than are most other synaptic proteins. (4) Biochemically, SCAMP 5 is highly enriched in purified synaptic vesicles. (5) Even neuroendocrine cells such as PC12 cells or chromaffin cells lack SCAMP 5, and chromaffin granules do not include this SCAMP isoform, revealing a high degree of specificity in the localization of this protein. Thus with SCAMP 5, we have identified a synaptic vesicle protein whose localization appears to be more specific for synaptic vesicles (as opposed to related secretory vesicles, e.g., secretory granules of neuroendocrine cells and synaptic-like microvesicles) than that of most other synaptic vesicle proteins.

Our analysis suggests, but does not conclusively prove, that SCAMPs 1 and 5 are the only SCAMP isoforms on synaptic vesicles. This would indicate that the lack of a major phenotype in the SCAMP 1 knock-out mice could be caused by functional redundancy of SCAMPs 1 and 5 in terms of a general "SCAMP function." However, SCAMP 5 lacks NPF repeats and cannot substitute for the proposed function of SCAMP 1 in binding EH-domain proteins during endocytosis (Fernández-Chacón et al., 2000). The endocytotic SCAMP 1 function may be redundant with synaptotagmin I because synaptotagmin has also been postulated to be a clathrin nucleation protein in endocytosis (Zhang et al., 1994). A further finding of our study is that a single cell line can express multiple SCAMPs, a finding that provides an additional rationale for the lack of a phenotype in the SCAMP 1 knock-out. It is intriguing that in the same cell, different SCAMPs may be differentially sorted as indicated by the presence of SCAMP 2 in adrenal medullary microsomes but its absence from chromaffin granules. This suggests that possibly even SCAMPs of the same NPF repeat-containing class may have distinct functions.

\section{Relation of SCAMPs to other proteins involved in membrane traffic with four TMRs}

It is striking that three separate families of proteins containing four TMRs are present on synaptic vesicles: SCAMPs, synaptophysins, and synaptogyrins (for review, see Fernández-Chacón and Südhof, 1999). All of these proteins exhibit the same transmembrane topology with cytoplasmic N- and C terminals. Synaptophysins and synaptogyrins constitute distinct protein families of highly homologous proteins that are, however, distantly related to each other 

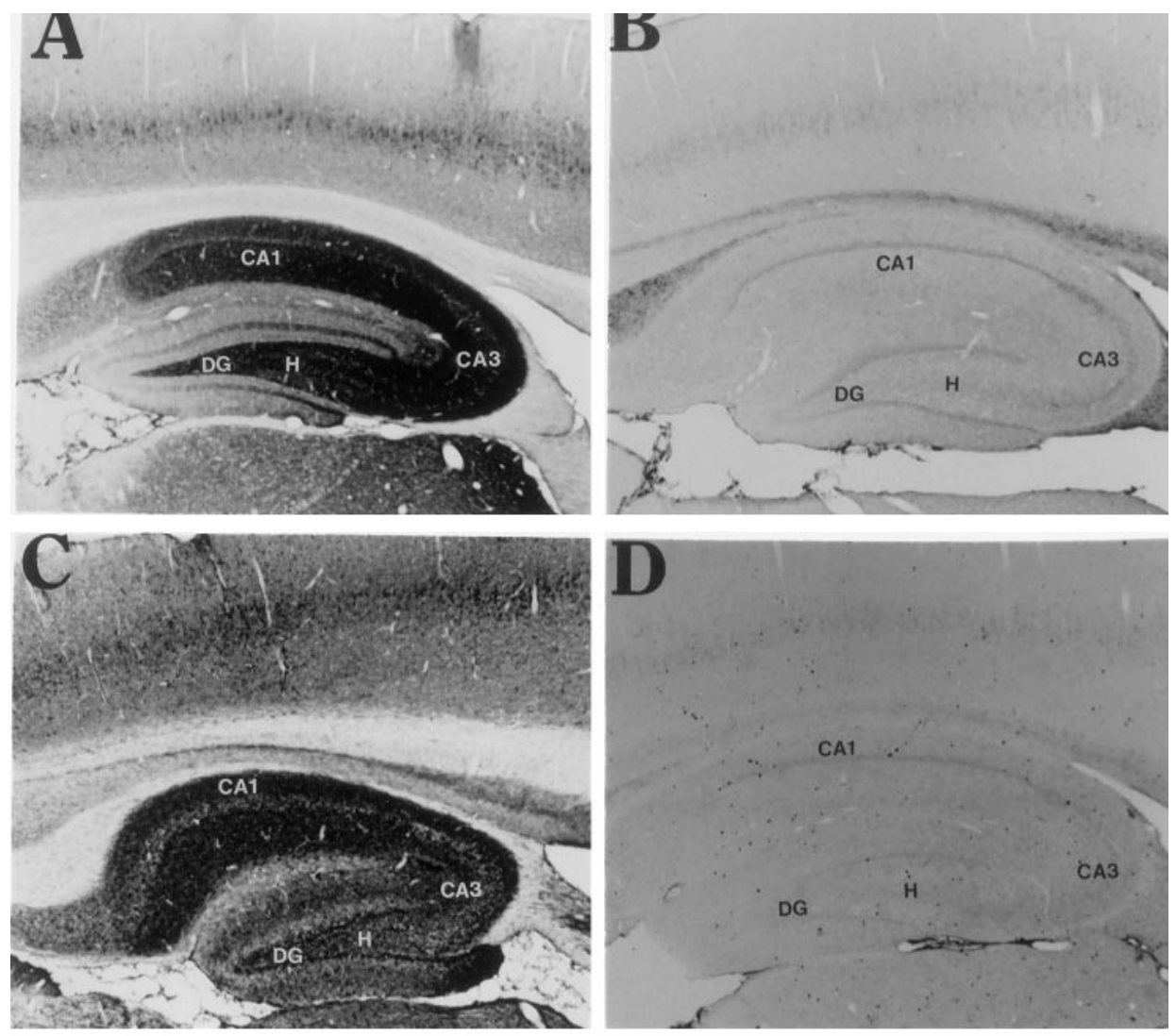

Figure 6. Localization of SCAMPs 1 and 5 in the rat hippocampus. $A-D$, Frozen sections of rat hippocampus were reacted with SCAMP 1 $(A, B)$ - and SCAMP $5(C, D)$-specific antibodies in the absence $(A, C)$ or presence $(B, D)$ of the antigen used to raise the antibody as a blocking agent of the specific signal. $E, F$, Sections were stained for synaptoporin and synapsins, respectively, as representative synaptic vesicle proteins. Immunoreactivity was visualized by HRP-heavy metal enhanced staining (Rosahl et al., 1995). The $C A 1$ and $C A 3$ regions, the hilus $(H)$, and the dentate gyrus $(D G)$ are identified by letters.
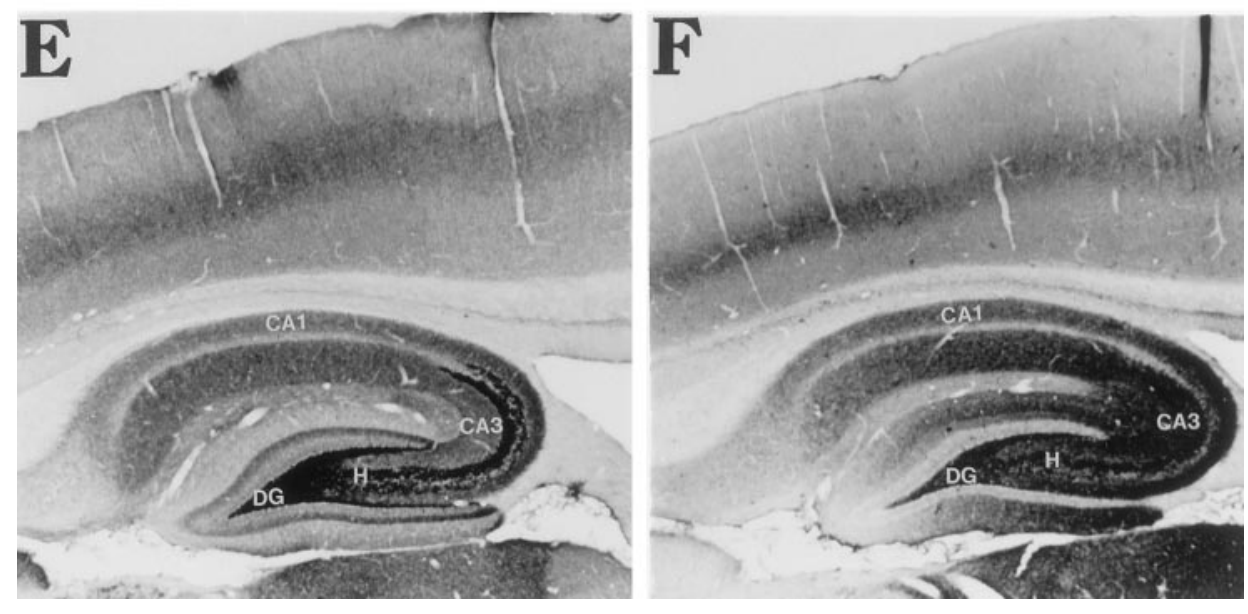

and exhibit functional redundancy (Janz et al., 1999; Sugita et al., 1999; for review, see Fernández-Chacón and Südhof, 1999). In contrast, the synaptophysins and synaptogyrins display no sequence homology to SCAMPs, except for an unusually high content of phenylalanine in the TMRs. All three protein families (SCAMPs, synaptophysins, and synaptogyrins) consist of ubiquitously distributed members in addition to members that are highly enriched in synaptic vesicles, or even specific for synaptic vesicles. Furthermore, at least some members of all three protein families are tyrosine phosphorylated (Baumert et al., 1990; Janz and Südhof, 1998; Wu and Castle, 1998). Members of all of these protein families are colocalized to trafficking vesicles in cells in general, and to synaptic vesicles in particular, and appear to recycle via the plasma membrane. This suggests that trafficking of recycling vesicles requires multiple proteins with four TMRs arranged in the same topology.

\section{Function of SCAMPs}

Our previous study had suggested that one of the functions of SCAMPs is to nucleate assembly of clathrin coats via binding of
EH-domain proteins to the N-terminal NPF repeats (FernándezChacón et al., 2000). Such a function would not necessarily require four TMRs, suggesting that the four TMRs in SCAMPs could be involved in an additional, possibly related function. This hypothesis is confirmed in the current experiments by the characterization of SCAMPs 4 and 5 as SCAMP isoforms that lack the $\mathrm{N}$-terminal NPF repeats but still include the highly conserved TMRs, suggesting that the NPF repeats are not central to SCAMP function. The fact that the TMRs in all of these proteins represent the most highly conserved sequences supports the idea that their functions directly involve the TMRs. It should be noticed that the novel vertebrate SCAMPs 4 and 5 described here appear to represent an evolutionary specialization. Data bank searches and cDNA sequencing revealed that invertebrates and plants express SCAMPs that also contain NPF repeats, whereas SCAMPs without NPF repeats were not found in these species. With the description of the plant and invertebrate SCAMPs, SCAMPs are among the most conserved membrane-trafficking proteins described.

We would like to argue that SCAMPs are probably multifunc- 

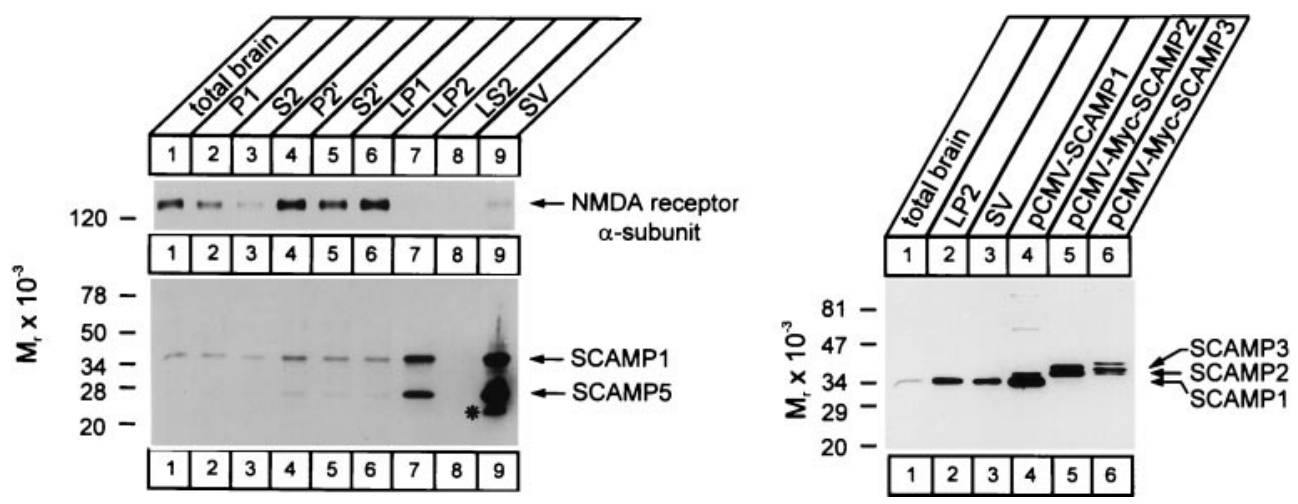

Figure 7. SCAMPs 1 and 5 are highly enriched on synaptic vesicles. $A$, Immunoblotting analysis of subcellular fractions from rat brain with antibodies to the NMDA receptor (top) or to SCAM Ps 1 and 5 (bottom). Total rat brain (lane 1) was subjected to standard low-speed and high-speed centrif ugations to yield a crude nuclear pellet $(P 1)$ and a synaptosomal pellet $\left(P 2^{\prime}\right)$. Synaptosomes were then subfractionated into heavy membranes $(L P 1)$, crude synaptic vesicles $(L P 2)$, and cytosol $(L S 2)$. Highly purified synaptic vesicles $(S V)$ were obtained from $L P 2$ fractions by controlled pore-glass chromatography. Note that the relative enrichment of SCAMP 5 with synaptic vesicles is greater than that of SCAMP 1 . The asterisk in lane 9 identifies a proteolytic breakdown product of SCAMPs. All lanes were loaded with $7.5 \mu \mathrm{g}$ of protein. $B$, Direct comparison of proteins that are immunoreactive with the SCAMP monoclonal antibody in total rat brain homogenates, in $L P 2$ and $S V$ fractions, and in COS cells transfected with SCAMPs 1-3 expression vectors. Note the cross-reactivity of the SCAMP monoclonal antibody under the conditions used here with multiple SCAMP isoforms expressed in COS cells. Numbers on the left indicate positions of molecular weight markers.

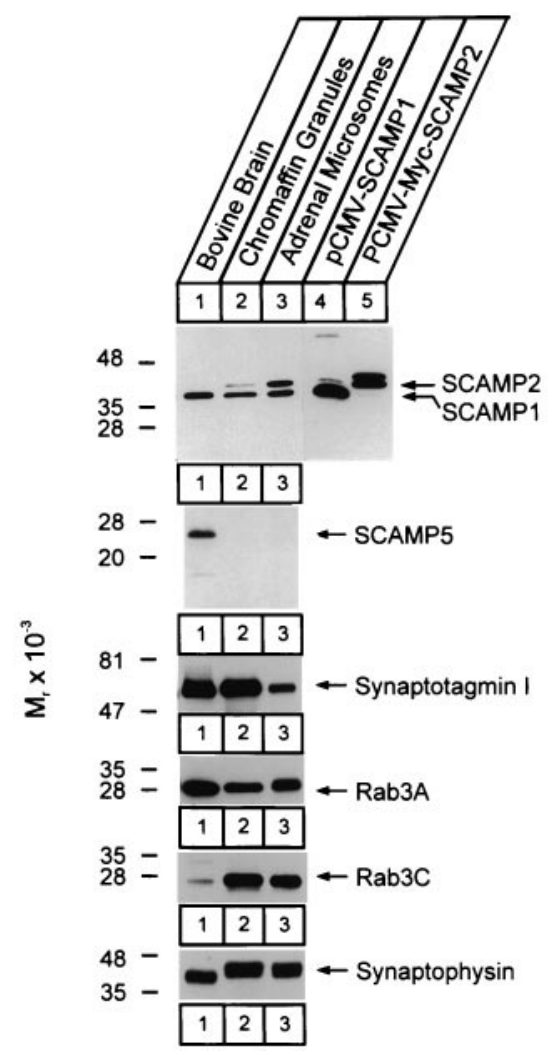

Figure 8. SCAMP 1 but not SCAMP 5 is enriched in bovine chromaffin granules. Total bovine brain homogenate (to ensure that the antibodies react with the bovine proteins; lane 1), purified chromaffin granules (lane 2 ), and adrenal microsomes (lane 3) were analyzed by immunoblotting with the SCAMP monoclonal antibody and with polyclonal antibodies to the various proteins indicated on the right. Only for the monoclonal SCAMP antibody (top; lanes 4, 5), transfected COS cells were also analyzed in parallel to identify the migration positions of SCAMPs 1 and 2. Numbers on the left indicate positions of molecular weight markers.

tional. The evidence of this hypothesis consists of the fact that we have defined two classes of SCAMPs that share most of their domains but differ in the presence or absence of the N-terminal NPF repeats. The NPF repeats probably recruit EH-domain proteins involved in clathrin-mediated budding events, a function that would not require four TMRs. At a minimum, SCAMPs probably perform two functions, one with the N-terminal NPF repeats in

clathrin-dependent budding and a second function with the conserved TMRs. The function of the TMRs is unknown in contrast to the NPF repeats, but their pattern of sequence conservation provides clues. It is interesting that the highest degree of conservation is found in the sequences on the membrane-cytosol interface (Fig. $1 A$ ). This finding indicates that the function of SCAMPs operates at this interface, with a most attractive role being in membrane fusion or fission that operates at this interface. Future experiments will have to address this issue.

\section{REFERENCES}

Altschul SF, Madden TL, Schäffer AA, Zhang J, Zhang Z, Miller W, Lipman DJ (1997) Gapped BLAST and PSI-BLAST: a new generation of protein database search programs. Nucleic Acids Res 25:3389-3402.

Baumert M, Takei K, Hartinger J, Burger PM, Fischer von Mollard G, Maycox PR, De Camilli P, Jahn R (1990) P29: a novel tyrosinephosphorylated membrane protein present in small clear vesicles of neurons and endocrine cells. J Cell Biol 110:1285-1294.

Brand SH, Castle JD (1993) SCAMP 37, a new marker within the general cell surface recycling system. EMBO J 12:3753-3761.

Brand SH, Laurie SM, Mixon MB, Castle JD (1991) Secretory carrier membrane proteins 31-35 define a common protein composition among secretory carrier membranes. J Biol Chem 266:18949-18957.

Butz S, Fernandez-Chacon R, Schmitz F, Jahn R, Südhof TC (1999) The subcellular localizations of atypical synaptotagmins: synaptotagmin III is enriched in synapses and synaptic plasma membranes but not in synaptic vesicles. J Biol Chem 274:18290-18296.

Cameron RS, Cameron PL, Castle JD (1986) A common spectrum of polypeptides occurs in secretion granule membranes of different exocrine glands. J Cell Biol 103:1299-1313.

De Beer T, Carter RE, Lobel-Rice KE, Sorkin A, Overduin M (1998) Structure and Asn-Pro-Phe binding of the Eps15 homology domain. Science 281:1357-1360.

Fazioli F, Minichiello L, Matoskova B, Wong WT, Di Fiore PP (1993) eps15, a novel tyrosine kinase substrate, exhibits transforming activity. Mol Cell Biol 13:5814-5828.

Fernández-Chacón R, Südhof TC (1999) Genetics of synaptic vesicle function: towards the complete functional anatomy of an organelle. Annu Rev Physiol 61:753-776.

Fernández-Chacón R, Alvarez de Toledo G, Hammer RE, Südhof TC (1999) Analysis of SCAMP1 function in secretory vesicle exocytosis by means of gene targeting in mice. J Biol Chem 274:32551-32554.

Fernández-Chacón R, Achiriloaie M, Janz R, Albanesi JP, Südhof TC (2000) SCAMP function in endocytosis. J Biol Chem 275:12752-12756.

Fischer von Mollard G, Mignery G, Baumert M, Perin MS, Hanson TJ, Burger PM, Jahn R, Südhof TC (1990) Rab3 is a small GTP-binding protein exclusively localized to synaptic vesicles. Proc Natl Acad Sci USA 87:1988-1992.

Gorman C (1985) High efficiency gene transfer into mammalian cells. In: DNA cloning, Vol II (Glover DM, ed), pp 143-190. Oxford: IRL.

Hussain NK, Yamabhai M, Ramjaun AR, Guy AM, Baranes D, O'Bryan JP, Der CJ, Kay BK, McPherson PS (1999) Splice variants of intersectin are components of the endocytic machinery in neurons and nonneuronal cells. J Biol Chem 274:15671-15677.

Huttner WB, Schiebler W, Greengard P, De Camilli P (1983) Synapsin I 
(protein I), a nerve terminal-specific phosphoprotein. III. Its association with synaptic vesicles studied in a highly purified synaptic vesicle preparation. J Cell Biol 96:1374-1388.

Janz R, Südhof TC (1998) Cellugyrin, a novel ubiquitous form of synaptogyrin that is phosphorylated by pp60-src. J Biol Chem 273:2851-2857.

Janz R, Südhof TC, Hammer RE, Unni V, Siegelbaum S, Bolshakov VY (1999) Essential roles in synaptic plasticity for synaptogyrin I and synaptophysin I. Neuron 24:687-700.

Johnston PA, Jahn R, Südhof TC (1989) Transmembrane topography and evolutionary conservation of synaptophysin. J Biol Chem 264:1268-1272.

Laemmli UK (1970) Cleavage of structural proteins during the assembly of the head of bacteriophage T4. Nature 227:680-685.

Laurie SM, Cain CC, Lienhard GE, Castle JD (1993) The glucose transporter GluT4 and secretory carrier membrane proteins (SCAMPs) colocalize in rat adipocytes and partially segregate during insulin stimulation. J Biol Chem 268:19110-19117.

Nagy A, Baker RR, Morris SJ, Whittaker VP (1976) The preparation and characterization of synaptic vesicles of high purity. Brain Res 109:285-309.

Okamoto M, Schoch S, Südhof TC (1999) EHSH1/intersectin, a protein that contains $\mathrm{EH}$ and $\mathrm{SH} 3$ domains and binds to dynamin and SNAP-25. A protein connection between exocytosis and endocytosis? J Biol Chem 274:18446-18454.

Page LJ, Sowerby PJ, Lui WW, Robinson MS (1999) $\gamma$-Synergin: an EH domain-containing protein that interacts with $\gamma$-adaptin. J Cell Bio 146:993-1004.

Paoluzi S, Castagnoli L, Lauro I, Salcini AE, Coda L, Fre' S, Confalonieri
S, Pelicci PG, Di Fiore PP, Cesareni G (1998) Recognition specificity of individual EH domains of mammals and yeast. EMBO J 17:6541-6550.

Rosahl TW, Spillane D, Missler M, Herz J, Selig DK, Wolff JR, Hammer RE, Malenka RC, Südhof TC (1995) Essential functions of synapsins I and II in synaptic vesicle regulation. Nature 375:488-493.

Sambrook J, Fritsch EF, Maniatis T (1989) Molecular cloning, a laboratory manual. Cold Spring Harbor, NY: Cold Spring Harbor Laboratory.

Sengar AS, Wang W, Bishay J, Cohen S, Egan SE (1999) The EH and SH3 domain Ese proteins regulate endocytosis by linking to dynamin and Eps15. EMBO J 18:1159-1171.

Singleton DR, Wu TT, Castle JD (1997) Three mammalian SCAMPs (secretory carrier membrane proteins) are highly related products of distinct genes having similar subcellular distributions. J Cell Sci 110:2099-2107.

Smith AD, Winkler H (1967) A simple method for the isolation of adrenal chromaffin granules on a large scale. Biochem J 103:480-482.

Sugita S, Janz R, Südhof TC (1999) Synaptogyrins regulate $\mathrm{Ca}^{2+}$ dependent exocytosis in PC12 cells. J Biol Chem 274:18893-18901.

Towbin H, Staehelin T, Gordon J (1979) Electrophoretic transfer of proteins from polyacrylamide gels to nitrocellulose sheets: procedure and some applications. Proc Natl Acad Sci USA 76:4350-4354.

Wu TT, Castle JD (1998) Tyrosine phosphorylation of selected secretory carrier membrane proteins, SCAMP1 and SCAMP3, and association with the EGF receptor. Mol Biol Cell 9:1661-1674.

Zhang JZ, Davletov BA, Südhof TC, Anderson RGW (1994) Synaptotagmin I is a high affinity receptor for clathrin AP2: implications for membrane recycling. Cell 78:751-760. 